\title{
Coverage Analysis for Millimeter Wave Cellular Networks with Beam Alignment Errors
}

\author{
${ }^{*}$ Ming Cheng, *Jun-Bo Wang, ${ }^{\dagger}$ Yongpeng Wu, ${ }^{\ddagger}$ Xiang-Gen Xia, \\ ${ }^{\S}$ Kai-Kit Wong, ${ }^{\top}$ Min Lin, ${ }^{*}$ Ming Chen \\ *National Mobile Communications Research Laboratory, Southeast University, 210096 Nanjing, China. \\ ${ }^{\dagger}$ Institute for Communications Engineering, Technical University of Munich, Theresienstrasse 90, D-80333 Munich, Germany. \\ $\ddagger$ Department of Electrical and Computer Engineering, University of Delaware, Newark, DE 19716 USA. \\ $\S$ Department of Electronic and Electrical Engineering, University College London, London WC1E 6BT, UK. \\ TCollege of Telecommunications and Information Engineering, Nanjing University of Posts and Telecommunications, 210003 \\ Nanjing, China. \\ Email: \{mingcheng, jbwang, chenming\}@seu.edu.cn, yongpeng.wu2016@gmail.com, xxia@ee.udel.edu, \\ kai-kit.wong@ucl.ac.uk, linmin@njupt.edu.cn.
}

\begin{abstract}
Millimeter wave (mmWave) communication is a promising approach to satisfy the demanding high data rate requirement of next generation mobile communications. This paper studies the downlink coverage performance of mmWave cellular networks with beam alignment errors. An enhanced antenna model is adopted to model the directional antenna beamforming pattern, in which the mainlobe beamwidth and directivity gains can be expressed as functions of the number of elements in the antenna array. After deriving the probability density function (PDF) of distance between mobile station (MS) and its serving base station (BS), the directivity gain with beam alignment errors is obtained as a discrete random variable. Then, a computationally tractable expression is derived for the coverage probability of mmWave cellular networks. Numerical results indicate that small beam alignment errors will not deteriorate the coverage probability significantly. Moreover, when the beam alignment error is small enough, the coverage performance can be improved by increasing the number of elements in the antenna array.
\end{abstract}

\section{INTRODUCTION}

With the fast development of portable devices and the radical increase of internet applications, mobile networks have become indispensable to our society. One of the main characteristics of future mobile networks is the unprecedented traffic volumes [1]. To cope with the exponentially increasing demands for high data rate wireless accesses, several key techniques have been proposed [2], [3]. In particular, millimeter wave (mmWave) communication, which benefits from its numerous spectrum resources and short wavelength, is widely considered as one of the most important technologies to achieve 10Gbit/s peak date rate. So far, many efforts have been devoted to the research of mmWave communications [4].

Thanks to the wider bandwidth and smaller wavelength compared to the sub- $6 \mathrm{GHz}$ signal utilized in conventional cellular networks, the mmWave signals can increase the system throughput effectively and efficiently. Large antenna arrays placed in a compact size can further provide high gains and directivities. However, the high frequency mmWave signals will experience severe path loss during the propagation, and suffer from severe penetration loss when they pass through common materials. Moreover, the severe penetration causes substantial difference between the line-of-sight (LOS) propagation paths and the non-line-of-sight (NLOS) propagation paths [5].

The system performance of mmWave communications has been studied in numerous exsiting works. Owing to the mathematical flexibility of stochastic geometry [6], there are several applications of stochastic geometry to study mmWave networks, such as analysis of coverage and capacity performance in cellular mmWave networks [7], [8] and in ad hoc mmWave networks [9], [10]. It should be noted that all studies in [7][10] characterized the impacts of beamforming of antenna arrays based on the flat-top model. The sinc and cosine antenna models were used to analyze the impacts of antenna array size on coverage performance in [11]. Unfortunately, since the antenna models in [11] were too complicated, the analysis was limited to the scenarios in which each mobile station (MS) is assumed to be equipped with only one single antenna. Moreover, the analyses in [7]-[11] assumed the beam alignment to be perfect which is impossible for practical systems. With imperfect beam alignment, the ergodic capacity for mmWave ad hoc networks was analyzed in [12]. However, the analytical expression for the ergodic capacity loss due to imperfect beam alignment in [12] was only valid in the high signal-to-interference-and-noise ratio (SINR) regime and the size of the antenna arrays has not been taken into account. To the best of our knowledge, there have been no published works which considered the impacts of both the number of elements in the antenna array and the beam alignment errors on the system performance of mmWave cellular communications.

This paper studies the downlink coverage performance for mmWave cellular networks with beam alignment errors. An enhanced flat-top antenna model is adopted to characterize the mainlobe beamwidth and directivity gains of antenna arrays as functions of the number of elements in the antenna array. Moreover, the directivity gains with imperfect beam alignment are derived as a discrete distributed random variables. By modeling the BSs as two independent nonhomogeneous Poisson point processes (PPPs), a computationally tractable analytical 
expression of downlink coverage probability is obtained for mmWave cellular networks.

\section{SySTEM MODEL}

\section{A. Network Model}

Consider a mmWave cellular network where all BSs are arranged according to a two dimensional homogenous PPP $\Phi$ with intensity $\lambda$. MSs are distributed as a stationary point process independent to the BSs. A typical MS, denoted as $\mathrm{MS}_{0}$, is assumed to be located at the origin $o$. All signals are transmitted using the mmWave bands. Due to the blockage effect in the propagation, the propagation path between BS and MS can be LOS or NLOS. Mathematically, the probability of being a LOS propagation path is

$$
\mathbb{P}_{\text {LOS }}(r)=e^{-\beta r},
$$

where $\beta$ is the blockage parameter determined by the density and average size of the blockages, and $r$ is the distance between the BS and the MS. Accordingly, the probability of a propagation path being NLOS is

$$
\mathbb{P}_{\text {NLOS }}(r)=1-\mathbb{P}_{\text {LOS }}(r) .
$$

The LOS probabilities are assumed to be independent for different BSs.

Based on the propagation paths to $\mathrm{MS}_{0}$, all BSs can be divided into two independent nonhomogenous PPPs, i.e., the LOS BS process $\Phi_{\mathrm{L}}$ with intensity function $\lambda \mathbb{P}_{\mathrm{LOS}}(r)$ and the NLOS BS process $\Phi_{\mathrm{N}}$ with intensity function $\lambda \mathbb{P}_{\text {NLOS }}(r)$. Furthermore, $\mathrm{MS}_{0}$ is served by the $\mathrm{BS}$, denoted as $\mathrm{BS}_{0}$, either LOS or NLOS, which provides the strongest average received power. In other words, the propagation path between $\mathrm{MS}_{0}$ and $\mathrm{BS}_{0}$ has the smallest path loss.

\section{B. Channel Model}

The LOS and NLOS propagation paths will have different path loss exponents, $\alpha_{\mathrm{L}}$ and $\alpha_{\mathrm{N}}$, respectively. In general, they satisfy $\alpha_{\mathrm{N}}>\alpha_{\mathrm{L}}>0$. Let the length of the propagation path between BS and MS be $r$, then the path loss $l(r)$ is described as

$$
l(r)=l_{\mathrm{L}}(r) \mathbb{1}_{\mathrm{LOS}}+l_{\mathrm{N}}(r) \mathbb{1}_{\mathrm{NLOS}}
$$

where $\mathbb{1}_{\text {LOS }}\left(\mathbb{1}_{\text {NLOS }}\right)$ is the Dirichlet function which is one when the BS is LOS (NLOS). $l_{\mathrm{L}}(r)$ and $l_{\mathrm{N}}(r)$ are path loss functions for LOS propagation path and NLOS propagation path, respectively. The two path loss functions are further assumed to be

$$
l_{\mathrm{L}}(r)=\left(1+r^{\alpha_{\mathrm{L}}}\right)^{-1}
$$

and

$$
l_{\mathrm{N}}(r)=\left(1+r^{\alpha_{\mathrm{N}}}\right)^{-1}
$$

respectively.

In this paper, the small scale fading on each propagation path is assumed to be independent Nakagami distributed. Then the power fading $h$ is a normalized Gamma random variable and its probability density function (PDF) is expressed as

$$
f_{h}(x)=\frac{m^{m}}{\Gamma(m)} x^{m-1} e^{-m x} \quad, \quad x>0,
$$

where $\Gamma(\cdot)$ is the Gamma function, and $m$ is the Nakagami parameter. For the LOS and NLOS propagation paths, $m$ is set to be $N_{\mathrm{L}}$ and $N_{\mathrm{N}}$, respectively. For the tractability of the following analysis, $N_{\mathrm{L}}$ and $N_{\mathrm{N}}$ are assumed to be positive integers [7].

\section{Enhanced Directional Beamforming Model}

In order to compensate for the frequency dependent pathloss, antenna arrays are assumed to be deployed at both the BSs and MSs to perform directional beamforming. To maintain the analytical tractability, the flat-top model is often used to characterize the pattern of the directional beamforming [13], which is shown in Fig. 1, where $G$ is the mainlobe directivity gain, $g$ is the sidelobe directivity gain and $\theta$ is the beamwidth of the mainlobe.

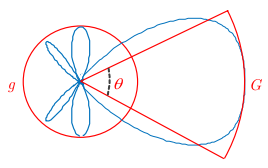

Fig. 1. Direction beamforming antenna model.

In this paper, antenna arrays deployed at both BSs and MSs are assumed to the uniform linear antenna arrays (ULAs) and we adopt a more realistic flat-top antenna model to depict the antenna radiation patterns. According to [14], for a ULA with $N$ antenna elements, if the maximum radiation intensity $U_{\max }$ is normalized to be one, the average intensity is approximated by

$$
U_{0} \approx \frac{\pi}{N k d}=\frac{\lambda_{\mathrm{c}}}{2 N d}
$$

where $\lambda_{c}$ is the wavelength, $d$ is the antenna element separation, and $k=2 \pi / \lambda_{\mathrm{c}}$ is the wave number. Meanwhile, the halfpower beamwidth (HPBW) can be expressed as [14]

$$
\theta_{\mathrm{H}} \simeq 2\left[\frac{\pi}{2}-\cos ^{-1}\left(\frac{1.391 \lambda_{c}}{\pi N d}\right)\right], \pi d / \lambda_{c} \ll 1 .
$$

If the antenna element separation is set to be half-wavelength, i.e., $d=\lambda_{c} / 2$, the radiation intensities and HPBW will be functions of the number of elements $N$ in the antenna array. Using (8), the mainlobe beamwidth in the flat-top model can be derived as

$$
\theta(N)=2 \theta_{\mathrm{H}}=2 \pi-4 \cos ^{-1}\left(\frac{2.782}{\pi N}\right) .
$$

If the mainlobe gain is assumed to be the maximum radiation intensity, i.e.

$$
G=U_{\max }=1
$$


the sidelobe gain will be a function of $N$ given by

$$
\begin{aligned}
g(N) & =\frac{2 \pi U_{0}-\theta(N) U_{\max }}{2 \pi-\theta} \\
& =\frac{(\pi / N)-\pi+2 \cos ^{-1}(2.782 /(\pi N))}{2 \cos ^{-1}(2.782 /(\pi N))} .
\end{aligned}
$$

\section{Beam Alignment Error Model}

In practice, implementation limitations will cause the antenna array point away from the desired target. In this paper, the beam alignment error $\delta$ is modeled as a truncated-Gaussian distributed variable with zero mean [12], whose PDF is

$$
f_{\delta}(t)=\frac{\sqrt{\frac{2}{\pi \sigma^{2}}} e^{-\frac{t^{2}}{2 \sigma^{2}}}}{\operatorname{erf}\left(\frac{\pi}{\sqrt{2} \sigma}\right)-\operatorname{erf}\left(\frac{-\pi}{\sqrt{2} \sigma}\right)}, \quad t \in(-\pi, \pi],
$$

where $\operatorname{erf}(x)=2 \int_{0}^{x} e^{-t^{2}} \mathrm{~d} t / \sqrt{\pi}$ is the error function, and $\sigma$ is the standard deviation of the original Gaussian variable. Furthermore, the expectation of the absolute error $|\delta|$ can be calculated by

$$
\mathbb{E}[|\delta|]=\frac{2 \sqrt{2} \sigma}{\operatorname{erf}\left(\frac{\pi-\mu}{\sqrt{2} \sigma}\right)-\operatorname{erf}\left(\frac{-\pi-\mu}{\sqrt{2} \sigma}\right)} \frac{1}{\sqrt{\pi}}\left(1-e^{-\frac{\pi^{2}}{2 \sigma^{2}}}\right) .
$$

According to (13), $|\delta|$ is a monotonically increasing function of $\sigma$.

\section{Downlink Coverage Analysis}

In this paper, all BSs are assumed to serve MSs with the same power. Mathematically, the coverage probability $P_{\mathrm{c}}$ is defined as the probability that the SINR at $\mathrm{MS}_{0}$ side is larger than some threshold $T$, i.e.,

$$
P_{\mathrm{c}} \triangleq \mathbb{P}[\mathrm{SINR}>T] \text {. }
$$

In (14), the received SINR can be expressed as

$$
\begin{aligned}
\mathrm{SINR} & \triangleq \frac{h_{0} m_{\mathrm{R}_{0}} m_{\mathrm{T}_{0}} l\left(r_{0}\right)}{\sum_{i \in \Phi \backslash\{0\}} h_{i} m_{\mathrm{R}_{i}} m_{\mathrm{T}_{i}} l\left(r_{i}\right)+\sigma_{n}^{2}} \\
& =\frac{h_{0} m_{\mathrm{R}_{0}} m_{\mathrm{T}_{0}} l\left(r_{0}\right)}{I_{\mathrm{L}}+I_{\mathrm{N}}+\sigma_{n}^{2}},
\end{aligned}
$$

where $h_{0}$ is the small scale fading on the desired propagation path, and $\sigma_{n}^{2}$ is the thermal noise power normalized by the transmit power, $m_{\mathrm{R}_{0}}\left(m_{\mathrm{R}_{i}}\right)$ is the directivity gain of receiving antenna array at $\mathrm{MS}_{0}$ for the desired (interfering) signal, $m_{\mathrm{T}_{0}}$ $\left(m_{\mathrm{T}_{i}}\right)$ is the directivity gain of transmitting antenna array at the serving $\mathrm{BS}_{0}$ (the interfering $\mathrm{BS}_{i}$ ), and $r_{0}\left(r_{i}\right)$ is the distance between $\mathrm{MS}_{0}$ and $\mathrm{BS}_{0}\left(\mathrm{BS}_{i}\right)$. In (15), $I_{\mathrm{L}}$ is the cumulative interference from all the other LOS BSs (except the serving $\mathrm{BS}$ for $\mathrm{MS}_{0}$ ) in $\Phi_{\mathrm{L}}$ and can be expressed as

$$
I_{\mathrm{L}}=\sum_{i \in \Phi_{\mathrm{L}} \backslash\{0\}} h_{\mathrm{L}_{i}} m_{\mathrm{R}_{i}} m_{\mathrm{T}_{i}} l_{\mathrm{L}}\left(r_{i}\right),
$$

where $h_{\mathrm{L}_{i}}$ is the small scale fading on the propagation path between $\mathrm{MS}_{0}$ and interfering LOS BS BS${ }_{i}$. Similarly, $I_{\mathrm{N}}$ is the cumulative interference from all the other NLOS BSs (except the serving $\mathrm{BS}$ for $\mathrm{MS}_{0}$ ) in $\Phi_{\mathrm{N}}$ and can be expressed as

$$
I_{\mathrm{N}}=\sum_{i \in \Phi_{\mathrm{N}} \backslash\{0\}} h_{\mathrm{N}_{i}} m_{\mathrm{R}_{i}} m_{\mathrm{T}_{i}} l_{\mathrm{N}}\left(r_{i}\right)
$$

where $h_{\mathrm{N}_{i}}$ is the small scale fading on the propagation path between $\mathrm{MS}_{0}$ and interfering NLOS BS BS${ }_{i}$.

\section{A. PDF of distance between $\mathrm{MS}_{0}$ and $\mathrm{BS}_{0}$}

Let $r_{\mathrm{L}}\left(r_{\mathrm{N}}\right)$ be the distance between $\mathrm{MS}_{0}$ and its nearest LOS (NLOS) BS. If the serving BS of $\mathrm{MS}_{0}$ is LOS, the path loss satisfies

$$
l_{\mathrm{L}}\left(r_{\mathrm{L}}\right)<l_{\mathrm{N}}\left(r_{N}\right),
$$

which can be derived as

$$
r_{\mathrm{N}}>r_{\mathrm{L}}^{\alpha_{\mathrm{L}} / \alpha_{\mathrm{N}}}
$$

Similarly, if the serving $\mathrm{BS}$ of $\mathrm{MS}_{0}$ is an NLOS one, we have

$$
r_{\mathrm{L}}>r_{\mathrm{N}}^{\alpha_{\mathrm{N}} / \alpha_{\mathrm{L}}}
$$

To facilitate the following analysis, two functions are defined as follows

$$
\begin{aligned}
& \psi_{\mathrm{L}}(r)=r^{\alpha_{\mathrm{L}} / \alpha_{\mathrm{N}}}, \\
& \psi_{\mathrm{N}}(r)=r^{\alpha_{\mathrm{N}} / \alpha_{\mathrm{L}}} .
\end{aligned}
$$

Lemma 1. If $\mathrm{MS}_{0}$ is associated with an LOS BS, the PDF of the distance to its serving $B S$ is

$$
\begin{aligned}
f_{\mathrm{L}}(x) & =2 \pi \lambda x \mathbb{P}_{\mathrm{LOS}}(x) \times \\
& e^{-2 \pi \lambda\left(\int_{0}^{x} t \mathbb{P}_{\mathrm{LOS}}(t) \mathrm{d} t+\int_{0}^{\psi_{\mathrm{L}}(x)} t \mathbb{P}_{\mathrm{NLOS}}(t) \mathrm{d} t\right)}, x>0 .
\end{aligned}
$$

In contrast, if $\mathrm{MS}_{0}$ is associated with an NLOS BS, the PDF of the distance to its serving $B S$ is

$$
\begin{aligned}
f_{\mathrm{N}}(x) & =2 \pi \lambda x \mathbb{P}_{\mathrm{NLOS}}(x) \times \\
& e^{-2 \pi \lambda\left(\int_{0}^{x} t \mathbb{P}_{\mathrm{NLOS}}(t) \mathrm{d} t+\int_{0}^{\psi_{\mathrm{N}}(x)} t \mathbb{P}_{\mathrm{LOS}}(t) \mathrm{d} t\right)}, x>0 .(24)
\end{aligned}
$$

Proof: The proof is given in Appendix A.

\section{B. Directivity Gains with Imperfect Beam Alignment}

Based on the enhanced flat-top beamforming model, the alignment is achieved when the azimuth of target transmitter or receiver falls in the mainlobe of its antenna array. In other words, if the absolute beam alignment error is not larger than half of the mainlobe beamwidth, i.e., $|\delta| \leq \theta(N) / 2$, the antenna array is deemed to be aligned. Using (12), the alignment probability can be calculated by

$$
\mathbb{P}_{\mathrm{A}}(\sigma, N)=\mathbb{P}\left[|\delta| \leq \frac{\theta(N)]}{2}\right]=\frac{\operatorname{erf}\left(\frac{\pi-2 \cos ^{-1}\left(\frac{2.782}{\pi N}\right)}{2 \sqrt{2} \sigma}\right)}{\operatorname{erf}\left(\frac{\pi}{\sqrt{2} \sigma}\right)}
$$

It can be observed from (25) that the alignment probability changes with $N$ and $\sigma$.

Since the beam alignment is not perfect, the directivity gain of the receiving (transmitting) antenna array for the desired signal of $\mathrm{MS}_{0}, m_{\mathrm{R}_{0}}\left(m_{\mathrm{T}_{0}}\right)$, can be described as a discrete 
random variable. Moreover, the probability mass functions (PMFs) of $m_{\mathrm{R}_{0}}$ and $m_{\mathrm{T}_{0}}$ can be expressed as

$$
f_{m_{\mathrm{R}_{0}}}(x)=\left\{\begin{array}{cc}
\mathbb{P}_{\mathrm{A}}\left(\sigma_{\mathrm{R}}, N_{\mathrm{R}}\right) & x=1 \\
1-\mathbb{P}_{\mathrm{A}}\left(\sigma_{\mathrm{R}}, N_{\mathrm{R}}\right) & x=g\left(N_{\mathrm{R}}\right)
\end{array}\right.
$$

and

$$
f_{m_{\mathrm{T}_{0}}}(x)=\left\{\begin{array}{cc}
\mathbb{P}_{\mathrm{A}}\left(\sigma_{\mathrm{T}}, N_{\mathrm{T}}\right) & x=1 \\
1-\mathbb{P}_{\mathrm{A}}\left(\sigma_{\mathrm{T}}, N_{\mathrm{T}}\right) & x=g\left(N_{\mathrm{T}}\right)
\end{array},\right.
$$

respectively, where $N_{\mathrm{R}}\left(N_{\mathrm{T}}\right)$ and $\sigma_{\mathrm{R}}\left(\sigma_{\mathrm{T}}\right)$ are the number of elements in the antenna array and the beam alignment error standard deviation of the receiving (transmitting) antenna array at MSs (BSs), respectively. Meanwhile, if both the AoAs and AoDs of interfering propagation paths are assumed to be independently and uniformly distributed in $(-\pi, \pi]$, the PMFs of directivity gains of the receiving and transmitting antenna arrays for interfering signals of $\mathrm{MS}_{0}, m_{\mathrm{R}_{i}}$ and $m_{\mathrm{T}_{i}}$, can be expressed as

$$
f_{m_{\mathrm{R}_{i}}}(x)=\left\{\begin{array}{cc}
\frac{\theta\left(N_{\mathrm{R}}\right)}{2 \pi} & x=1 \\
1-\frac{\theta\left(N_{\mathrm{R}}\right)}{2 \pi} & x=g\left(N_{\mathrm{R}}\right)
\end{array}\right.
$$

and

$$
f_{m_{\mathrm{T}_{i}}}(x)=\left\{\begin{array}{cc}
\frac{\theta\left(N_{\mathrm{T}}\right)}{2 \pi} & x=1 \\
1-\frac{\theta\left(N_{\mathrm{T}}\right)}{2 \pi} & x=g\left(N_{\mathrm{T}}\right)
\end{array},\right.
$$

respectively.

\section{Coverage Analysis}

Denote the set of events that $\mathrm{MS}_{0}$ is covered by an MS as $\mathcal{A}$. Thus, $\mathcal{A}$ can be divided into two disjoint subsets, $\mathcal{A}_{\mathrm{L}}$ and $\mathcal{A}_{\mathrm{N}}$, which are the sets of events that $\mathrm{MS}_{0}$ is covered by an LOS MS and an NLOS MS, respectively. Accordingly, the coverage probability $P_{\mathrm{c}}$ can be expressed as

$$
\begin{aligned}
P_{\mathrm{c}} & =P_{\mathcal{A}}=P_{\mathcal{A}_{\mathrm{L}}}+P_{\mathcal{A}_{\mathrm{N}}} \\
& =\int_{0}^{\infty} P_{\mathrm{c}, \mathrm{L}}(x) f_{\mathrm{L}}(x) \mathrm{d} x+\int_{0}^{\infty} P_{\mathrm{c}, \mathrm{N}}(x) f_{\mathrm{N}}(x) \mathrm{d} x,(30)
\end{aligned}
$$

where $f_{\mathrm{L}}(x)$ and $f_{\mathrm{N}}(x)$ are the PDFs given in Lemma 1, $P_{\mathrm{c}, \mathrm{L}}(x)\left(P_{\mathrm{c}, \mathrm{N}}(x)\right)$ is the conditional coverage probability given the condition that $\mathrm{MS}_{0}$ is served by an LOS (NLOS) BS located at distance of $x$.

Theorem 1. If $\mathrm{MS}_{0}$ is served by an LOS BS or an NLOS BS located at distance of $x$, the conditional coverage probabilities can be respectively obtained in (31) and (32) at the top of the next page, where $s_{\mathrm{L}}(x)=\frac{T \beta_{\mathrm{L}}}{m_{\mathrm{R}_{0}} m_{\mathrm{T}_{0}} l_{\mathrm{L}}(x)}, s_{\mathrm{N}}(x)=$ $\frac{T \beta_{\mathrm{N}}}{m_{\mathrm{R}_{0}} m_{\mathrm{T}_{0}} l_{\mathrm{N}}(x)}, \quad \beta_{\mathrm{R}}=N_{\mathrm{R}}\left(N_{\mathrm{R}} !\right)^{-\frac{1}{N_{\mathrm{R}}}}, \beta_{\mathrm{T}}=N_{\mathrm{T}}\left(N_{\mathrm{T}} !\right)^{-\frac{1}{N_{\mathrm{T}}}}$. $\mathcal{L}_{I_{\mathrm{L}, \mathrm{LOS}}}(s)$ and $\mathcal{L}_{I_{\mathrm{N}, \mathrm{LOS}}}(s)\left(\mathcal{L}_{I_{\mathrm{L}, \mathrm{NLOS}}}(s)\right.$ and $\left.\mathcal{L}_{I_{\mathrm{N}, \mathrm{NLOS}}}(s)\right)$ are the Laplace transforms (LTS) of interference $I_{\mathrm{L}}$ and $I_{\mathrm{N}}$ with respect to $s$ under the condition that $\mathrm{MS}_{0}$ is served by an LOS (NLOS) BS, respectively.

Proof: The proof is given in Appendix B.

The derivations of the LTs of $I_{\mathrm{L}, \mathrm{LOS}}, I_{\mathrm{N}, \mathrm{LOS}}, I_{\mathrm{L}, \mathrm{NLOS}}$ and $I_{\mathrm{N}, \mathrm{NLOS}}$ are given in Appendix C.

Using (23), (24), (26)-(29), (C.1)-(C.4), the coverage probability of $\mathrm{MS}_{0}$ can be further derived in Theorem 2 .
Theorem 2. In the mmWave cellular network with imperfect alignment, if $\mathrm{MS}_{0}$ is served by the $B S$ which provides the largest received signal power, the coverage probability $P_{\mathrm{c}}$ can be obtained in (33) at the top of the next page.

Proof: (31) can be obtained by substituting (31) and (32) into (30) and changing the order of the integer and the summation.

\section{Numerical Results}

In this section, the impacts of alignment errors and the number of elements in the antenna array on the system coverage probability will be discussed, and the accuracy of coverage probability expression will be verified. Without loss of generality, the mmWave cellular network is assumed to work in an environment with blockage parameter $\beta=0.0069$ such that the BS at the distance of 100 meters can be LOS and NLOS with equal probability. The BS intensity is assumed to be $\lambda=\frac{1}{\pi} \times 10^{-4} \mathrm{~m}^{-2}$. The SINR threshold is set to be $T=10 \mathrm{~dB}$. The noise power normalized by the transmit power is set to be $-124 \mathrm{~dB}$. Referring to the parameters setting in [7], the path loss exponents and Nakagami parameters are set to be $\alpha_{\mathrm{L}}=2\left(\alpha_{\mathrm{N}}=4\right)$ and $N_{\mathrm{L}}=3\left(N_{\mathrm{N}}=2\right)$ for LOS (NLOS) propagation paths, respectively. Due to the space limit of MS, the number of elements in the antenna array at MS is set to be $N_{\mathrm{R}}=4$.

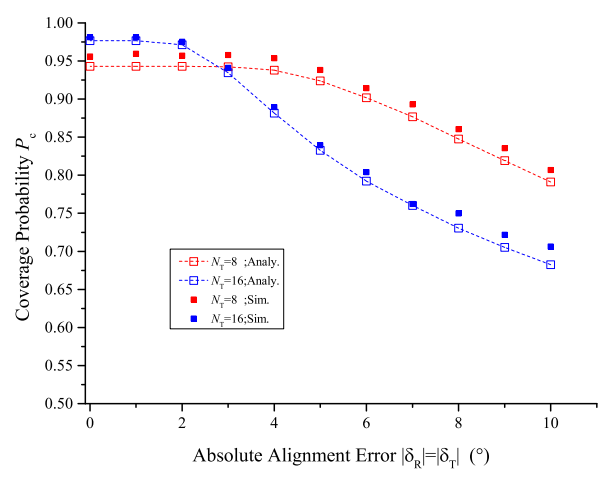

Fig. 2. Coverage probability versus $|\delta|$ with $\left|\delta_{\mathrm{R}}\right|=\left|\delta_{\mathrm{T}}\right|=|\delta|$.

Fig. 2 provides the coverage probability with different beam alignment errors. It can be seen that the coverage probability decreases with the increase of alignment errors. Moreover, when the alignment error is relatively small, the decrease of the coverage probability is not remarkable. Particularly, it can be seen that the maxima of average absolute alignment errors without degrading the coverage performance are $4^{\circ}$ and $2^{\circ}$ for $N_{\mathrm{T}}=8$ and $N_{\mathrm{T}}=16$, respectively. This is because that when the average absolute alignment error is smaller than half of the mainlobe beamwidth, the misalignment occurs with low probability.

Fig. 3 shows the coverage probability with different number of elements in the antenna array. It can be seen that when the number of elements in the antenna array is small, the coverage probability can be improved by adding antenna elements. The 


$$
\begin{aligned}
& P_{\mathrm{c}, \mathrm{L}}=\mathbb{E}_{m_{\mathrm{R}_{0}}, m_{\mathrm{T}_{0}}}\left[\sum_{n=1}^{N_{\mathrm{L}}}(-1)^{n+1}\left(\begin{array}{l}
N_{\mathrm{L}} \\
n
\end{array}\right) e^{-n s_{\mathrm{L}}(x) \sigma_{n}^{2}} \mathcal{L}_{I_{\mathrm{L}, \mathrm{LOS}}}\left(n s_{\mathrm{L}}(x)\right) \mathcal{L}_{I_{\mathrm{N}, \mathrm{LOS}}}\left(n s_{\mathrm{L}}(x)\right)\right] \\
& P_{\mathrm{c}, \mathrm{N}}=\mathbb{E}_{m_{\mathrm{R}_{0}}, m_{\mathrm{T}_{0}}}\left[\sum_{n=1}^{N_{\mathrm{N}}}(-1)^{n+1}\left(\begin{array}{l}
N_{\mathrm{N}} \\
n
\end{array}\right) e^{-n s_{\mathrm{N}}(x) \sigma_{n}^{2}} \mathcal{L}_{I_{\mathrm{L}, \mathrm{NLOS}}}\left(n s_{\mathrm{N}}(x)\right) \mathcal{L}_{I_{\mathrm{N}, \mathrm{NLOS}}}\left(n s_{\mathrm{N}}(x)\right)\right]
\end{aligned}
$$

$$
\begin{aligned}
P_{\mathrm{c}} & =\sum_{n=1}^{N_{\mathrm{L}}}(-1)^{n+1}\left(\begin{array}{l}
N_{\mathrm{L}} \\
n
\end{array}\right) \int_{0}^{\infty} f_{\mathrm{L}}(x) \mathbb{E}_{m_{\mathrm{R}_{0}}, m_{\mathrm{T}_{\mathrm{O}}}}\left[e^{-n s_{\mathrm{L}}(x) \sigma_{n}^{2}} \mathcal{L}_{I_{\mathrm{L}, \mathrm{LOS}}}\left(n s_{\mathrm{L}}(x)\right) \mathcal{L}_{I_{\mathrm{N}, \mathrm{LOS}}}\left(n s_{\mathrm{L}}(x)\right)\right] \mathrm{d} x \\
& +\sum_{n=1}^{N_{\mathrm{N}}}(-1)^{n+1}\left(\begin{array}{l}
N_{\mathrm{N}} \\
n
\end{array}\right) \int_{0}^{\infty} f_{\mathrm{N}}(x) \mathbb{E}_{m_{\mathrm{R}_{0}}, m_{\mathrm{T}_{\mathrm{O}}}}\left[e^{-n s_{\mathrm{N}}(x) \sigma_{n}^{2}} \mathcal{L}_{I_{\mathrm{L}, \mathrm{NLOS}}}\left(n s_{\mathrm{N}}(x)\right) \mathcal{L}_{I_{\mathrm{N}, \mathrm{NLOS}}}\left(n s_{\mathrm{N}}(x)\right)\right] \mathrm{d} x
\end{aligned}
$$

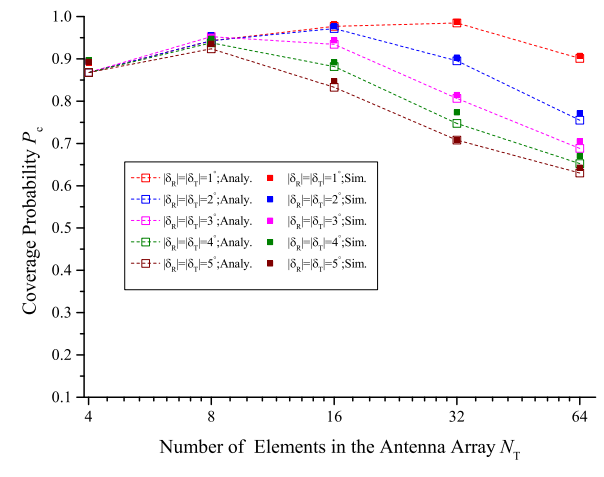

Fig. 3. Coverage probability versus $N_{\mathrm{T}}$ for different beam alignment errors.

reason is that the mainlobe beamwidth of small antenna array is wide enough to keep beam alignment. Therefore, interference power can be decreased by reducing the sidelobe gain of the antenna array. However, it should be noted that increasing the number of elements in the antenna array cannot always improve the coverage probability. It can be observed that when the number of elements in the antenna array grows larger and the alignment errors exist, the mainlobe beamwidth becomes too narrow to guarantee the beam alignment. Therefore, the coverage probability deteriorates significantly.

\section{CONClusion}

This paper analyzes the coverage probability of mmWave celluar networks with beam alignment errors. Based on the enhanced flat-top antenna model, we analyze the impacts of number of elements in the antenna array and the beam alignment errors on the coverage performance. The coverage probability is derived in a tractable analytical expression. Simulation results verify the accuracy of our theoretical analysis. Numerical results show that the coverage performance will not be deteriorated by small beam alignment errors and when the beam alignment errors are small enough, the coverage performance can be improved by increasing the number of elements in the antenna array. However, when the beam alignment errors are large, the alignment probability of large antenna arrays may not high enough such that increasing the number of elements in the antenna array will decrease the coverage probability.

\section{ACKNOWLEDGMENTS}

This work is supported by the National Nature Science Foundation of China (No.61571115) and the European Union's Horizon 2020 research and innovation programme under the Marie Skłodowska-Curie grant agreement (No.709291).

\section{APPENDiX A}

\section{PROOF OF LEMMA 1}

The probability that $\mathrm{MS}_{0}$ is served by an LOS BS at distance of $x$ can be obtained as

$$
\begin{aligned}
P_{\mathrm{L}} & =e^{-2 \pi \lambda\left(\int_{0}^{x} t \mathbb{P}_{\mathrm{LOS}}(t) \mathrm{d} t+\int_{0}^{\psi_{L}(x+\Delta x)} t \mathbb{P}_{\mathrm{NLOS}}(t) \mathrm{d} t\right)} \\
& \times\left(1-e^{-2 \pi \lambda \int_{x}^{x+\Delta x} t \mathbb{P}_{\mathrm{LOS}}(t) \mathrm{d} t}\right), x>0, \Delta x \rightarrow 0 .
\end{aligned}
$$

Then, the PDF of the distance between $\mathrm{MS}_{0}$ and its serving LOS BS can be obtained as

$$
\begin{aligned}
f_{\mathrm{L}}(x) & =\lim _{\Delta x \rightarrow 0} \frac{P_{\mathrm{L}}}{\Delta x}=2 \pi \lambda x \mathbb{P}_{\mathrm{LOS}}(x) \times \\
& e^{-2 \pi \lambda\left(\int_{0}^{x} t \mathbb{P}_{\mathrm{LOS}}(t) \mathrm{d} t+\int_{0}^{\psi_{\mathrm{L}}(x)} t \mathbb{P}_{\mathrm{NLOS}}(t) \mathrm{d} t\right)}{ }^{\prime} x>0 .(\text { A. } 2)
\end{aligned}
$$

The derivation of $f_{\mathrm{N}}(x)$ is in the similar manner to $f_{\mathrm{L}}(x)$.

\section{APPENDIX B \\ PROOF OF THEOREM 1}

If $\mathrm{MS}_{0}$ is served by an LOS BS located at the distance of $x$, the received SINR at $\mathrm{MS}_{0}$ is

$$
\begin{aligned}
& \mathrm{SINR}_{\mathrm{L}} \\
& =\frac{h_{\mathrm{L} 0} m_{\mathrm{R}_{0}} m_{\mathrm{T}_{0}} l_{\mathrm{L}}(x)}{\sum_{i \in \Phi_{\mathrm{L}} \backslash b(o, x)} h_{\mathrm{L} i} m_{\mathrm{R}_{i}} m_{\mathrm{T}_{i}} l_{\mathrm{L}}\left(r_{i}\right)+\sum_{i \in \Phi_{\mathrm{N}} \backslash b\left(o, \psi_{\mathrm{L}}(x)\right)} h_{\mathrm{Ni}} m_{\mathrm{R}_{i}} m_{\mathrm{T}_{i}} l_{\mathrm{N}}\left(r_{i}\right)+\sigma_{n}^{2}} \\
& =\frac{h_{\mathrm{L} 0} m_{\mathrm{R}_{0}} m_{\mathrm{T}_{0}} l_{\mathrm{L}}(x)}{I_{\mathrm{L}, \mathrm{LOS}}+I_{\mathrm{N}, \mathrm{LOS}}+\sigma_{n}^{2}} .
\end{aligned}
$$




$$
\begin{aligned}
P_{\mathrm{c}, \mathrm{L}} & =\mathbb{P}\left[\mathrm{SINR}_{\mathrm{L}}>T\right]=\mathbb{P}\left[\frac{h_{\mathrm{L} 0} m_{\mathrm{R}_{0}} m_{\mathrm{T}_{0}} l_{\mathrm{L}}(x)}{I_{\mathrm{L}, \mathrm{LOS}}+I_{\mathrm{N}, \mathrm{LOS}}+\sigma_{n}^{2}}>T\right]=\mathbb{P}\left[h_{\mathrm{L} 0}>\frac{T}{m_{\mathrm{R}_{0}} m_{\mathrm{T}_{0}} l_{\mathrm{L}}(x)}\left(I_{\mathrm{L}, \mathrm{LOS}}+I_{\mathrm{N}, \mathrm{LOS}}+\sigma_{n}^{2}\right)\right] \\
& \stackrel{(a)}{\approx} \mathbb{E}_{m_{\mathrm{R}_{0}}, m_{\mathrm{T}_{0}}}\left[1-\mathbb{E}_{I_{\mathrm{L}, \mathrm{LOS}}, I_{\mathrm{N}, \mathrm{LOS}}}\left[\left(1-e^{-\frac{m^{m}}{m_{\mathrm{R}_{0}} \mathrm{~T}_{0} l_{\mathrm{L}}(x)}(x)\left(I_{\mathrm{L}, \mathrm{LOS}}+I_{\mathrm{N}, \mathrm{LOS}}+\sigma_{n}^{2}\right)}\right)^{N_{\mathrm{L}}}\right]\right] \\
& \stackrel{(b)}{=} \mathbb{E}_{m_{\mathrm{R}_{0}}, m_{\mathrm{T}_{0}}}\left[\sum_{n=1}^{N_{\mathrm{L}}}(-1)^{n+1}\left(\begin{array}{l}
N_{\mathrm{L}} \\
n
\end{array}\right) \mathbb{E}_{I_{\mathrm{L}, \mathrm{LOS}}, I_{\mathrm{N}, \mathrm{LOS}}}\left[e^{-n s_{\mathrm{L}}(x)\left(I_{\mathrm{L}, \mathrm{LOS}}+I_{\mathrm{N}, \mathrm{LOS}}+\sigma_{n}^{2}\right)}\right]\right] \\
& \stackrel{(c)}{=} \mathbb{E}_{m_{\mathrm{R}_{0}}, m_{\mathrm{T}_{0}}}\left[\sum_{n=1}^{N_{\mathrm{L}}}(-1)^{n+1}\left(\begin{array}{l}
N_{\mathrm{L}} \\
n
\end{array}\right) e^{-n s_{\mathrm{L}}(x) \sigma_{n}^{2}} \mathcal{L}_{I_{\mathrm{L}, \mathrm{LOS}}}\left(n s_{\mathrm{L}}(x)\right) \mathcal{L}_{I_{\mathrm{N}, \mathrm{LOS}}}\left(n s_{\mathrm{L}}(x)\right)\right]
\end{aligned}
$$

The conditional coverage probabilities $P_{\mathrm{c}, \mathrm{L}}$ can be calculated by (B.2) at the top of this page, where $(a)$ is from [15] and the independence between the directivity gains and the point process of the BSs, $(b)$ follows the Binomial theorem and the assumption that $N_{\mathrm{L}}$ is an integer, $(c)$ follows from the independence between $\Phi_{\mathrm{L}}$ and $\Phi_{\mathrm{N}}$, and the definition of the LT. $P_{\mathrm{c}, \mathrm{N}}$ can be derived in the similar manner to $P_{\mathrm{c}, \mathrm{L}}$.

\section{APPENDIX C \\ DERIVATIONS OF THE LTS}

The LT of $I_{\mathrm{L}, \mathrm{LOS}}$ can be calculated as

$$
\begin{aligned}
& \mathcal{L}_{I_{\mathrm{L}, \mathrm{LOS}}}(s)=\mathbb{E}\left[e^{-s \sum_{i \in \Phi_{\mathrm{L}} \backslash b(o, x)} h_{\mathrm{Li}} m_{\mathrm{R}_{i}} m_{\mathrm{T}_{i}} l_{\mathrm{L}}\left(r_{i}\right)}\right] \\
& \stackrel{(a)}{=} \mathbb{E}\left[\prod_{i \in \Phi_{\mathrm{L}} \backslash b(o, x)} e^{\left.-s h_{\mathrm{L} i} m_{\mathrm{R}_{i}} m_{\mathrm{T}_{i}} l_{\mathrm{L}}\left(r_{i}\right)\right]}\right. \\
& \stackrel{(b)}{=} e^{-2 \pi \lambda \mathbb{E}_{m_{\mathrm{R}_{i}}, m_{\mathrm{T}_{i}}}\left[\int _ { x } ^ { \infty } \left(1-h_{\mathrm{L}}\left[e^{\left.\left.\left.-s l_{\mathrm{L}}(t) h_{\mathrm{L}} m_{\mathrm{R}_{i}} m_{\mathrm{T}_{i}}\right]\right) t \mathbb{P}_{\mathrm{LOS}}(t) \mathrm{d} t\right]}\right.\right.\right.} \\
& \stackrel{(c)}{=} e^{-2 \pi \lambda \mathbb{E}_{m_{\mathrm{R}_{i}}, m_{\mathrm{T}_{i}}}\left[\int_{x}^{\infty}\left(1-\left(\frac{1}{1+\frac{s l_{\mathrm{L}}(t) m_{\mathrm{R}_{i}} m_{\mathrm{T}_{i}}}{N_{\mathrm{L}}}}\right)^{N_{\mathrm{L}}}\right) t \mathbb{P}_{\mathrm{LOS}}(t) \mathrm{d} t\right]},(\mathrm{C} .1)
\end{aligned}
$$

where $(a)$ follows the independence between different interfering LOS propagation paths, $(b)$ follows the probability generating functional (PGFL) of the PPP, and $(c)$ is from the moment generating function of $h_{\mathrm{L}}$. Similarly, the LTs of $I_{\mathrm{N}, \mathrm{LOS}}, I_{\mathrm{L}, \mathrm{NLOS}}$ and $I_{\mathrm{N}, \mathrm{NLOS}}$ can be derived as follows.

$$
\begin{aligned}
& \mathcal{L}_{I_{\mathrm{N}, \mathrm{LOS}}}(s)= \\
& e^{-2 \pi \lambda \mathbb{E}_{m_{\mathrm{R}_{i}}, m_{\mathrm{T}}}\left[\int_{\psi_{\mathrm{L}}(x)}^{\infty}\left(1-\left(\frac{1}{1+\frac{s l_{\mathrm{N}}(t) m_{\mathrm{R}_{i}} m_{\mathrm{T}_{i}}}{N_{\mathrm{N}}}}\right)^{N \mathrm{M}}\right) t \mathbb{P}_{\mathrm{NLOS}}(t) \mathrm{d} t\right]} \\
& \mathcal{L}_{I_{\mathrm{L}, \mathrm{NLOS}}}(s)= \\
& e^{-2 \pi \lambda \mathbb{E}_{m_{\mathrm{R}_{i}}, m_{\mathrm{T}_{i}}}\left[\int_{\psi_{\mathrm{N}}(x)}^{\infty}\left(1-\left(\frac{1}{1+\frac{s l_{\mathrm{L}}(t) m_{\mathrm{R}_{i}} m_{\mathrm{T}_{i}}}{N_{\mathrm{L}}}}\right)^{N_{\mathrm{L}}}\right) t \mathbb{P}_{\mathrm{LOS}}(t) \mathrm{d} t\right]} \\
& \mathcal{L}_{I_{\mathrm{N}, \mathrm{NLOS}}}(s)= \\
& e^{-2 \pi \lambda \mathbb{E}_{m_{\mathrm{R}_{i}}, m_{\mathrm{T}_{i}}}\left[\int_{x}^{\infty}\left(1-\left(\frac{1}{1+\frac{s l_{\mathrm{N}}(t) m_{\mathrm{R}_{i}} m_{\mathrm{T}_{i}}}{N_{\mathrm{N}}}}\right)^{N_{\mathrm{M}}}\right) t \mathbb{P}_{\mathrm{NLOS}}(t) \mathrm{d} t\right]},
\end{aligned}
$$

respectively.

\section{REFERENCES}

[1] J. G. Andrews, S. Buzzi, W. Choi, S. V. Hanly, A. Lozano, A. C. K. Soong, and J. C. Zhang, "What will 5G be?" IEEE J. Sel. Areas Commun., vol. 32, no. 6, pp. 1065-1082, Jun. 2014.

[2] A. Osseiran, F. Boccardi, V. Braun, K. Kusume, P. Marsch, M. Maternia, O. Queseth, M. Schellmann, H. Schotten, H. Taoka, H. Tullberg, M. A. Uusitalo, B. Timus, and M. Fallgren, "Scenarios for 5G mobile and wireless communications: The vision of the METIS project," IEEE Commun. Mag., vol. 52, no. 5, pp. 26-35, May 2014.

[3] C. X. Wang, F. Haider, X. Gao, X. H. You, Y. Yang, D. Yuan, H. M. Aggoune, H. Haas, S. Fletcher, and E. Hepsaydir, "Cellular architecture and key technologies for 5G wireless communication networks," IEEE Commun. Mag., vol. 52, no. 2, pp. 122-130, Feb. 2014.

[4] Z. Pi and F. Khan, "An introduction to millimeter-wave mobile broadband systems," IEEE Commun. Mag., vol. 49, no. 6, pp. 101-107, Jun. 2011.

[5] S. Rajagopal, S. Abu-Surra, and M. Malmirchegini, "Channel feasibility for outdoor Non-line-of-sight mmWave mobile communication," in Proc. IEEE VTC Fall, Québec City, Canada, Sept. 2012, pp. 1-6.

[6] F. Baccelli and B. Błszczyszyn, "Stochastic Geometry and Wireless Networks: Volume I Theory," Foundations and Trends in Networking, vol. 3, no. 1-2, pp. 249-449, 2010

[7] T. Bai and R. W. Heath, "Coverage and rate analysis for millimeter-wave cellular networks," IEEE Trans. Wireless Commun., vol. 14, no. 2, pp. 1100-1114, Feb. 2015

[8] L. Wang, K. K. Wong, R. W. Heath, and J. Yuan, "Wireless powered dense cellular metworks: How many small cells do we need?" IEEE J. Sel. Areas Commun., vol. PP, no. 99, pp. 1-1, 2017.

[9] A. Thornburg, T. Bai, and R. W. Heath, "Performance analysis of outdoor mmWave ad hoc networks," IEEE Trans. Sign. Proc., vol. 64, no. 15, pp. 4065-4079, Aug. 2016.

[10] Y. Zhu, L. Wang, K. K. Wong, and R. W. Heath, "Secure communications in millimeter wave ad hoc networks," IEEE Trans. Wireless Commun., vol. 16, no. 5, pp. 3205-3217, May 2017.

[11] X. Yu, J. Zhang, M. Haenggi, and K. B. Letaief, "Coverage analysis for millimeter wave networks: The impact of directional antenna arrays," IEEE J. Sel. Areas Commun., vol. PP, no. 99, pp. 1-1, Apr. 2017.

[12] A. Thornburg and R. W. Heath, "Ergodic capacity in mmWave ad hoc network with imperfect beam alignment," in Proc. IEEE MILCOM, Tampa, FL, USA, Oct. 2015, pp. 1479-1484.

[13] A. M. Hunter, J. G. Andrews, and S. Weber, "Transmission capacity of ad hoc networks with spatial diversity," IEEE Trans. Wireless Commun., vol. 7, no. 12, pp. 5058-5071, Dec. 2008.

[14] C. A. Balanis, Antenna Theory: Analysis and Design (4th Edition). John Wiley and Sons Inc., 2016.

[15] H. Alzer, "Some inequalities for the incomplete Gamma function," Math. Comput., vol. 66, no. 218, pp. 771-778, 1997. 